\title{
An Iterative Self-Absorption Correction Algorithm for 3D Ptycho-Fluorescence Imaging.
}

$\underline{\text { Silvia Cipiccia }}{ }^{1, *}$, Darren J. Batey ${ }^{1}$, Xiaowen Shi $^{1}$, Stephen Price ${ }^{1}$, Aaron Parsons ${ }^{1}$, Kaz Wanelik ${ }^{1}$, Andrew Wilson ${ }^{1}$, Robert Crook $^{1}$, Robert Raja ${ }^{2}$ and Christoph Rau ${ }^{1,3,4}$

1. Diamond Light Source, Harwell Science and Innovation Campus, Didcot OX11 0QX, UK

2. University of Southampton, University Road, Southampton, SO17 1BJ, UK

3. University of Manchester, Manchester M1 7HS, UK

4. Northwestern University, Feinberg School of Medicine, Chicago, Illinois 60611, USA

* Corresponding author, silvia.cipiccia@diamond.ac.uk

We present an iterative self-absorption correction algorithm developed in the coherence branch of I13 beamline at Diamond light source, based on simultaneous 3-D fluorescence and ptychography acquisition.

$\mathrm{X}$-ray micro-fluorescence is a powerful nondestructive imaging technique to access the chemical composition of samples. The x-ray fluorescence tomography requires absorption correction when the self-absorption of the sample is not negligible. Schroer in 2001[1] tackled the problem calculating the mass absorption coefficient at the fluorescence energies from the asymmetry of the fluorescence sinogram. Alternatively the mass absorption coefficients have been estimated from combining fluorescence, transmission and Compton tomography[2]. Based on Schroer study, Huang in 2017 [3] presented an iterative self-absorption correction algorithm for quantitative $\mathrm{x}$-ray fluorescence computed tomography.

Fluorescence microscopy has been coupled to x-ray ptychography [4] to achieve high resolution chemical and structural information. Ptychography is a coherent small-angle scattering technique. In xray ptychography, the x-ray beam is focused onto a sample so that a small area of the sample is illuminated. The sample is then scanned through the x-ray beam in a series of overlapping illuminated areas. The diffraction pattern of each area is recorded and then processed with an iterative algorithm. Due to the redundancy of information provided by the overlapping areas, the ptychography reconstruction algorithm is robust in solving the phase problem and capable of retrieving both the object and the probe phase and modulus. Ptychography experiments are routinely performed in I13 coherence branch[5].

The algorithm presented here combines the absorption information retrieved with the ptychography technique and the fluorescence signal acquired with a detector placed at 90 degrees from the propagation of the beam (Figure 1). The starting point of the algorithm is the 3D object modulus reconstructed from the ptycho-tomography and the initial 3D chemical element distribution obtained from the uncorrected sinograms. From the initial guess, the effective density of the elements is calculated per each pixel and the fluorescence sinograms corrected for self-absorption. Once corrected, the sinograms are used to produce a new 3D distribution and recalculate the effective density from the 3D absorption ptychotomography. The algorithm has been applied to fluorescence signal detected during ptycho-tomography scan of a catalytic sample. The sample contained platinum nanoparticles nested in copper chlorophosphate framework. The open-framework structure similarly consists of $\mu^{4}$ chloride ions at the apex of $4 \times \mathrm{CuO}_{4} \mathrm{Cl}$ square-based pyramids. Pyrophosphate $\left(\mathrm{P}_{2} \mathrm{O}_{7}\right)$ units link these quartets forming 
analogous 1D channels. The $\left[\mathrm{CuO}_{4}\right]_{4} \mathrm{Cl}\left[\mathrm{P}_{2} \mathrm{O}_{7}\right]_{4}$ units are cross-linked via a shared pyrophosphate oxygen atom in the ab plane by square-based $\mathrm{CuO}_{4}$ groups. This produces the square grid of $1 \mathrm{D}$ channels approx. $12.7 \AA$ in diameter.

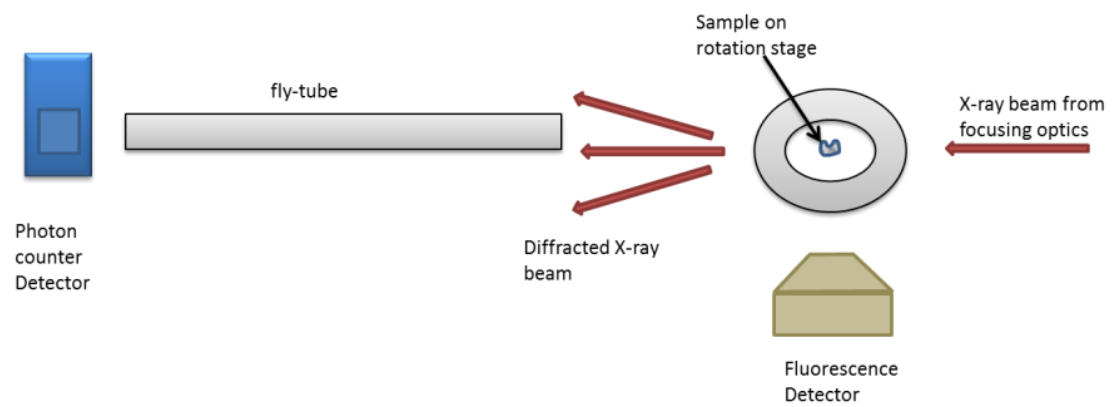

Figure. 1. Experimental setup. The x-ray beam is focused using a Fresnel zone plate. The beam size at the sample is about $600 \mathrm{~nm}$. The fluorescence signal is collected at 90 degrees from the direction of propagation of the x-ray beam. The ptychography detector is located along the x-ray beam about $2 \mathrm{~m}$ after the sample. The results presented here are not deconvolved by the beam size. However, the achievable resolution is given by $250 \mathrm{~nm}$ the step size [6] in 2D, and it is estimated to be less than $500 \mathrm{~nm}$ in 3D.

The channels are occupied by charge compensating $\mathrm{Rb}^{+}, \mathrm{K}^{+}$or $\mathrm{NH}_{4}^{+}$cations and the templating tetrachloro metallate anions such as $\left[\mathrm{AuCl}_{4}\right]^{-},\left[\mathrm{PdCl}_{4}\right]^{2-}$ or $\left[\mathrm{PtCl}_{4}\right]^{2-}$. Figure 2 shows a reconstructed tomography slice of copper signal at different algorithm iterations. Figure 3 shows in detail the effect of the algorithm on the value of the copper signal in a pixel [7].

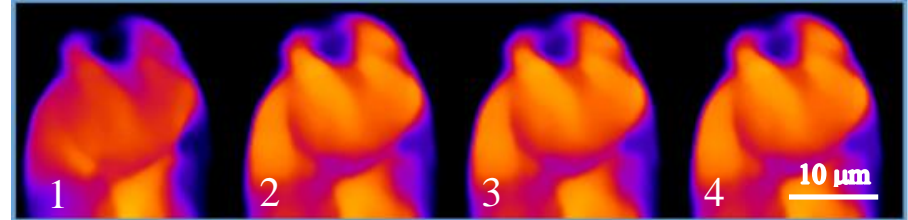

Figure. 2. A slice of the tomography reconstructed copper signal for four consecutive iterations.

[1] C. G. Schroer, Applied Physics Letters 79,12(2001), p. 1912

[2] B. Golosio et al, Journal of Applied physics 94(2003), p.145

[3] R. Huang et al, AIP Advances 7 (2017), p. 05511

[4] D. J. Vine et al., Opt. Express 20 (2012) 18287

[5] D. J. Batey, et al., Physical Review A 89 (2014), p. 043812.

[6] S. Ehn et al., Biomed Opt Express 7,4 (2016), p1227-1239.

[7] We would like to thank Dr Matthew E. Potter, Dr Arran M Gill and Mr Jack D. Parsons for the sample preparation

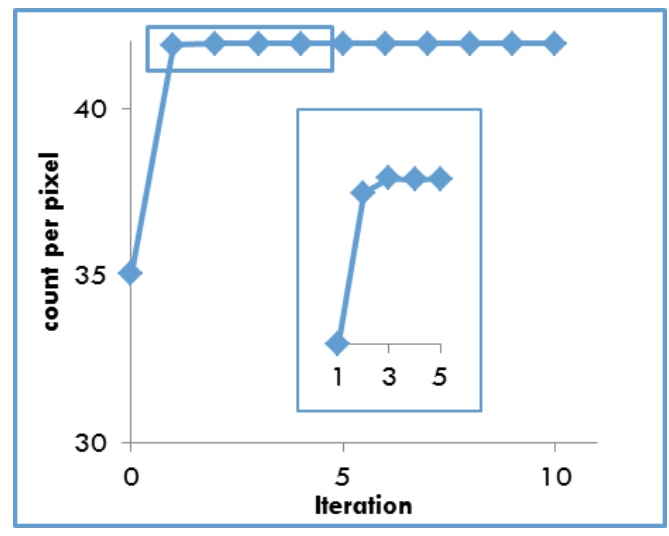

Figure. 3. Effect of the iterative algorithm on the signal in a pixel for the copper signal. 